Clinical Vistas

\section{Transfusion-related acute}

\section{lung injury}

$\infty \quad$ See related article page I4I

$\mathrm{A}$ 67-year-old man with diabetes mellitus, chronic cystitis and hematuria was given 2 units of packed red blood cells because of severe iron-deficiency anemia (hemoglobin $54 \mathrm{~g} / \mathrm{L}$ ). Although he was pale, a physical examination on admission revealed normal vital signs. Following the transfusion, the patient was feeling well, and his vital signs were unchanged. Forty minutes after the second unit of red blood cells was transfused, the patient experienced a sudden onset of dyspnea, tachypnea (34 breaths/min), cyanosis, fever, profuse diaphoresis and hypoxemia (oxygen saturation $<75 \%$ ). The patient's blood pressure was $150 / 80 \mathrm{~mm} \mathrm{Hg}$, and his temperature was $38.7^{\circ} \mathrm{C}$. His breath sounds were markedly decreased, and chest auscultation revealed bilateral diffuse crackles. There were no signs of volume overload, and his jugular venous pressure was normal. An electrocardiogram appeared normal, and the test results for tro$\stackrel{\circ}{\circ}$ ponin and kidney function were within the normal range. A chest radiograph showed coarse alveolar infiltrates and a normal cardiac silhouette (Figure I), which is the radiographic hallmark of noncardiogenic pulmonary edema. A CT scan showed diffuse edema and inflammatory consolidation of both lungs (Figure 2). The patient remained unstable, with a urine output of $250 \mathrm{~mL}$ in the 5 -hour period after diuretics were administered; nitroglycerin and steroids were given, and mechanical ventilation was started. Fluid was not aspirated from the patient's lungs when his trachea was intubated. The patient's condition slowly improved, and he achieved a full recovery over the next 6 days. Blood transaminase levels peaked on the second day (alanine transaminase II23 U/L; aspartate transaminase $875 \mathrm{U} / \mathrm{L}$ ) and returned to normal on the fourth day; bilirubin levels remained normal. Six days later, both a chest radiograph and a CT scan showed the patient's lungs to be clear. The plasma from the 2 donors and the recipient was not assayed for anti-human leukocyte antigen (HLA) or anti-leukocyte antibodies. HLA typing of the recipient was not performed.

Transfusion-related acute lung injury (TRALI) is a rare, yet severe and po- tentially fatal, adverse event that affects about I in 5000 patients who receive transfusions. In most cases the reaction occurs within 30 minutes to 2 hours, although it may occur up to 6 hours post transfusion. This type of transfusion reaction is characterized by the sudden onset of respiratory failure, severe hypoxemia and pulmonary edema (shown by radiography) despite normal cardiac function. ${ }^{1}$ In the present case, other potential causes of respiratory distress that could have been triggered by the transfusion (e.g., volume overload, anaphylaxis, hemolysis, transfusion of blood products contaminated with bacteria) were ruled out on the basis of the patient's history and presentation as well as by the clinical features and laboratory findings.

The pathogenesis of transfusionrelated acute lung injury is unclear. Anti-HLA and anti-neutrophil antibodies transferred from the donor to the recipient are thought to bind to and activate blood leukocytes, which then become sequestered in the lungs where they cause damage to the endothelium and parenchyma, leakage of fluid into the alveoli and alveolar inflammation. ${ }^{2}$ Alternatively, donor leukocytes may be activated by recipient-specific antibodies. Another theory suggests that 2 subsequent, independent events are re-

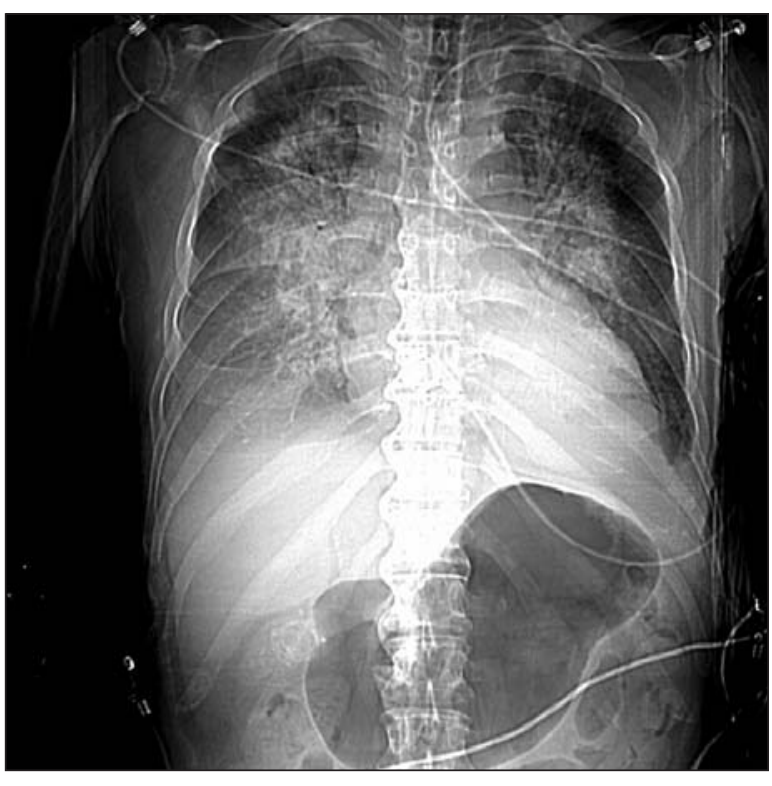

Figure 1: A chest radiograph showing coarse alveolar infiltrates and a normal cardiac silhouette.

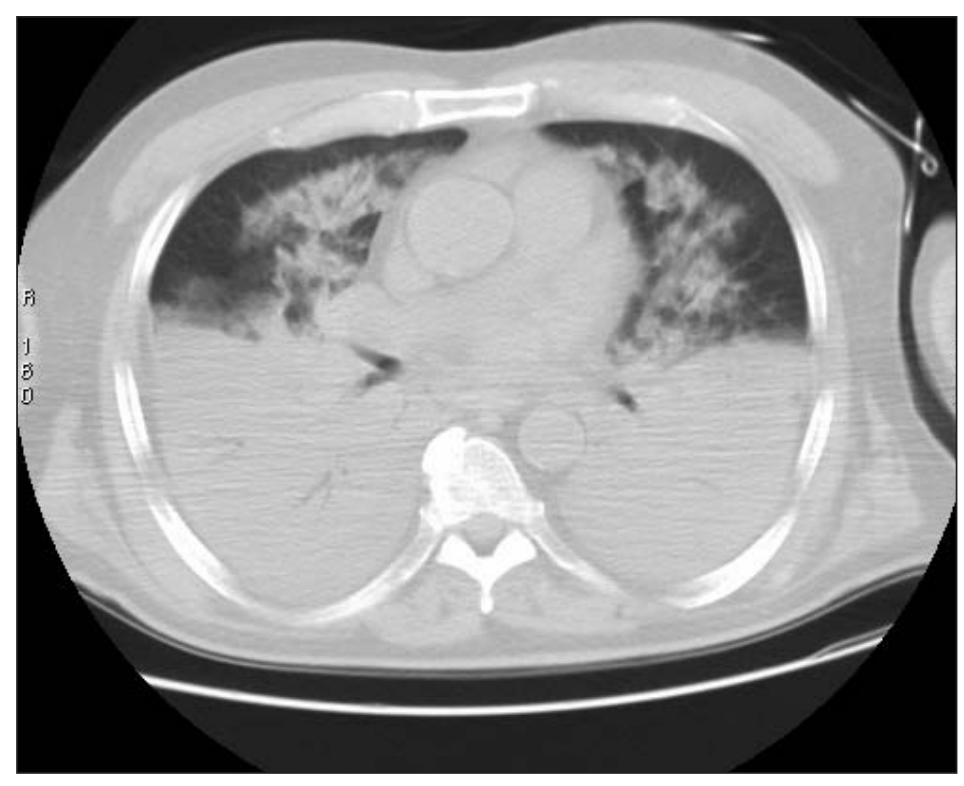

Figure 2: A CT scan showing diffuse edema and inflammatory consolidation of both lungs. 
quired for the pathogenesis of transfusion-related acute lung injury: the endothelial activation and sequestration of neutrophils in the lungs, ${ }^{1}$ and the release of enzymes and reactive-oxygen species by primed leukocytes, triggered by passively transfused anti-HLA or anti-neutrophil antibodies. This "2-hit" hypothesis suggests that patients with underlying systemic inflammation, such as those undergoing major surgery, those with severe viral or bacterial infections, those given massive transfusions or cytokines and those receiving bone marrow or solid organ transplants, may be at increased risk of transfusion-related acute lung injury. ${ }^{1}$

Any blood product that contains plasma has the potential to cause a transfusion-related acute lung injury; however, platelet concentrates derived from whole blood are most commonly implicated, followed by fresh-frozen plasma, packed red blood cells, whole blood, granulocytes, cryoprecipitate and intravenous immunoglobulin. ${ }^{1}$

Our patient experienced a transfusionrelated acute lung injury after the transfusion of 2 units of buffy-coatdepleted "young" packed red bood cells (from a 30-year-old man and a 58-year-old man) that were drawn 7 days earlier. This indirectly rules out the possibility that the "second hit" was due to lipid pro-inflammatory mediators accumulating during blood storage because of aging of erythrocytes and leukocytes. Furthermore, our patient had not received any prior transfusions, which reduces the chance of previous sensitization against major histocompatibilitycomplex antigens.

Transfusion-related acute lung injury is diagnosed by the recognition of clinical symptoms and signs; serologic studies are not needed except in rare cases. Treatment is primarily supportive, and mechanical ventilation is often required for several days. Mortality is estimated to be about $5 \%$; however, most survivors recover their baseline lung function without further sequelae. We postulate that the elevated blood transaminase levels in our patient's case reflect the diffuse inflammatory damage to the lungs rather than liver injury.

\section{Giuseppe Famularo MD PhD}

Department of Internal Medicine

Michele Galluzzo MD

Department of Radiology

San Camillo Hospital

Rome, Italy

This article has been peer reviewed.

Competing interests: None declared.

\section{REFERENCES}

I. Silliman CC, Ambruso DR, Boshkov LK. Transfusion-related acute lung injury. Blood 2005;105: 2266-73.

2. Kopko PM, Popovsky MA, MacKenzie MR, et al. HLA class II antibodies in transfusion-related acute lung injury. Transfusion 200I;4I:I244-8. 